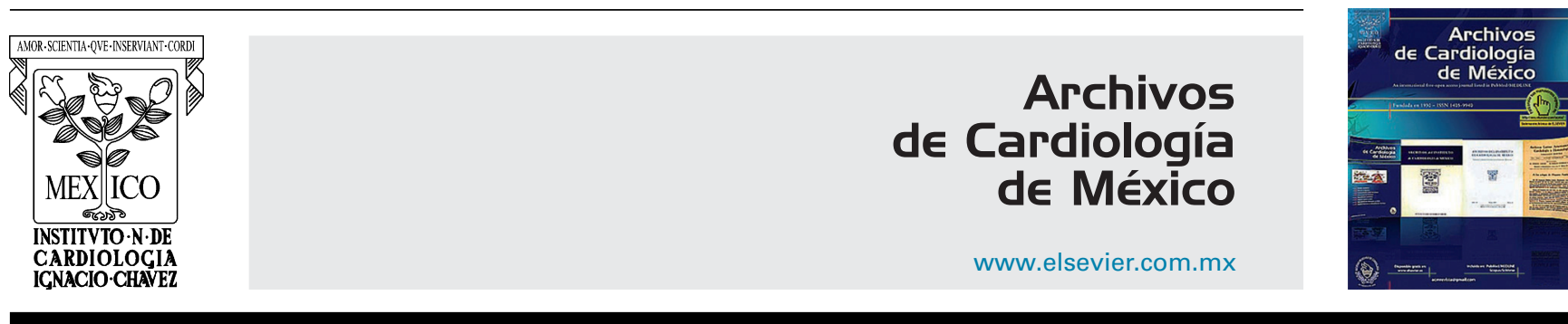

INVESTIGACIÓN CLÍNICA

\title{
Efectos de la estimulación endocárdica prolongada sobre la sincronía mecánica ventricular izquierda. Ensayo piloto aplicando análisis de fase por tomografía computada por emisión de fotón único gatillada. Estimulación prolongada y disincronía mecánica
}

\author{
Federico Ferrando-Castagnetto ${ }^{\mathrm{a}, \mathrm{b}, *}$, Roberto Ricca-Mallada ${ }^{\mathrm{a}}$, \\ Alejandro Vidal ${ }^{\mathrm{a}}$ y Rodolfo Ferrando ${ }^{\mathrm{b}}$
}

\footnotetext{
a Departamento de Cardiología-Centro Cardiovascular Universitario, Hospital de Clínicas, Facultad de Medicina, Universidad de la República, Montevideo, Uruguay

b Servicio de Medicina Nuclear-Centro Asistencial del Sindicato Médico, Montevideo, Uruguay
}

Recibido el 5 de agosto de 2016; aceptado el 19 de diciembre de 2016

\section{PALABRAS CLAVE \\ Disincronía; \\ Marcapasos; \\ Infarto; \\ Uruguay}

\begin{abstract}
Resumen
Objetivo: Evaluar la disincronía mecánica ventricular izquierda (DMVI) asociada a la estimulación endocárdica prolongada del ventrículo derecho mediante el ensayo piloto de una nueva técnica de imagen en Uruguay.

Métodos: Se estudiaron 12 pacientes con marcapasos implantados al menos hace un año utilizando SPECT gatillada con ${ }^{99 \mathrm{~m}} \mathrm{Tc}-\mathrm{MIBI}$ y análisis de fase en reposo. Se registraron variables clínicas, duración del QRS, tasa, modo y sitio de estimulación ventricular, presencia y extensión de infarto e isquemia, volúmenes cavitarios y FEVI en reposo. Utilizando V-Sync de Emory Cardiac Toolbox se obtuvieron desviación estándar y ancho de banda de fase en reposo (grados), comparando los individuos con $\mathrm{FEVI} \geq \mathrm{y}<50 \%$ con una población control.

Resultados: Los pacientes de la serie con estimulación endocárdica prolongada en el ventrículo derecho exhibieron marcada DMVI. Los pacientes con FEVI moderada/severamente disminuida (con diámetros cavitarios mayores, estimulación septal, infarto extenso o isquemia significativa) exhibieron mayor disincronía que aquellos con FEVI conservada/levemente disminuida (ancho de banda $=144.33^{\circ}$ vs. $77.41^{\circ}, p<0.05$; desviación estándar $=46.67^{\circ}$ vs. $26.81^{\circ}, p<0.05$ ).
\end{abstract}

\footnotetext{
* Autor para correspondencia. Hospital de Clínicas p. 2, Avenida Italia s/n. Teléfono/fax: (+00598) 24871515

Correo electrónico: federico.ferrando@gmail.com (F. Ferrando-Castagnetto).
} 
Conclusiones: En la cardiopatía isquémica la elevada tasa de estimulación endocárdica crónica se asocia con marcada DMVI, aun cuando la función sistólica esté conservada. El análisis de fase podría ser una herramienta válida para evaluar la disincronía asociada al infarto en pacientes con marcapasos, y aportar a la indicación oportuna del pasaje (upgrading) al modo de estimulación biventricular.

(C) 2016 Instituto Nacional de Cardiología Ignacio Chávez. Publicado por Masson Doyma México S.A. Este es un artículo Open Access bajo la licencia CC BY-NC-ND (http://creativecommons. org/licenses/by-nc-nd/4.0/).

\section{KEYWORDS}

Dyssynchrony; Pacemaker; Infarct; Uruguay
Effects of prolonged endocardial stimulation on left ventricular mechanical synchrony. A pilot study applying gated-SPECT phase analysis. Endocardial stimulation and dyssynchrony

\begin{abstract}
Objetive: To evaluate left ventricular mechanical dyssynchrony (LVMD) associated with prolonged right ventricular pacing through an innovative imaging technique as a pilot study in Uruguay.

Methods: A 99mTc-MIBI gated-SPECT and phase analysis was performed in 12 patients with pace-makers implanted at least one year before scintigraphy, due to advanced atrioventricular block. Clinical data, QRS duration, rate, mode and site of pacing in right ventricle, chamber diameters, presence and extension of myocardial scar and ischaemia, as well as LVEF at rest, were recorded. Using V-Sync of Emory Cardiac Toolbox, a standard deviation (PSD) and bandwidth (PBW) from rest phase histogram was obtained and these indexes were compared with controls in the subgroups of patients with LVEF $\geq 50 \%$ and $<50 \%$.

Results: Patients with prolonged RV endocardial pacing exhibited marked LVMD. More severe dyssynchrony was found in patients with impaired LVEF than in patients with preserved LVEF (PSD: $46.67^{\circ}$ vs. $26.81^{\circ}, P<.05$; PBW: $144.33^{\circ}$ vs. $\left.77.41^{\circ}, P<.05\right)$. Higher left ventricle diameters, extensive infarct, or significant ischaemia were found in patients with impaired LVEF.

Conclusions: Chronic right ventricular pacing was invariably associated with LVMD, even when systolic function was preserved. Phase analysis could be a potentially useful technique to evaluate LMVD associated with myocardial scar in patients with pacemakers, and to decide promptly the upgrading to biventricular pacing.

(C) 2016 Instituto Nacional de Cardiología Ignacio Chávez. Published by Masson Doyma México S.A. This is an open access article under the CC BY-NC-ND license (http://creativecommons. org/licenses/by-nc-nd/4.0/).
\end{abstract}

\section{Introducción}

La terapia con marcapasos es de elección en la disfunción sinusal y el bloqueo auriculoventricular avanzado. Desafortunadamente, sus beneficios se obtienen a expensas de una secuencia de activación ventricular izquierda muy anómala.

En el bloqueo completo de la rama izquierda (BCRI) la activación del ventrículo izquierdo (VI) determina una relajación septal anormal que pierde energía para el desarrollo de las fuerzas laterales y, a la vez, determina un cierre mitral subóptimo ${ }^{1}$; ambos eventos disminuyen considerablemente la capacidad eyectiva del $\mathrm{Vl}^{2}$. Este comportamiento contráctil descoordinado (disincrónico) se observa tanto en el BCRI nativo ${ }^{3}$ como en la estimulación endocárdica del ventrículo derecho (VD) mediante marcapasos ${ }^{4}$. En esta última la disincronía mecánica intraventricular izquierda (DMVI) se asocia con daños estructurales tempranos, como disrupción miofibrilar, fibrosis, remodelado anómalo, alteraciones metabólicas y en la perfusión ${ }^{2,5}$. A largo plazo tiende a disminuir la función contráctil, dilatar el VI, deteriorar los síntomas, desarrollar fibrilación auricular y disminuir la sobrevida ${ }^{6-8}$. Como estos efectos clínicos y funcionales son difícilmente predecibles, persisten controversias acerca de las indicaciones del pasaje (upgrading) a la terapia de resincronización cardíaca (TRC) en los pacientes con marcapasos que desarrollan disfunción sistólica severa.

$\mathrm{El}$ análisis de fase (AF) por SPECT gatillada es una técnica de reciente desarrollo que evalúa la DMVI de forma válida y confiable ${ }^{9}$. Sin embargo, los aportes de esta técnica al estudio de la mecánica contráctil en sujetos portadores de marcapasos han sido escasamente documentados. Nuestra experiencia se dirigió a caracterizar la DMVI asociada a la estimulación endocárdica prolongada del VD en una serie de casos portadores de cardiopatía isquémica, aplicando el AF a modo de ensayo piloto.

\section{Métodos}

Se estudiaron los pacientes portadores de marcapasos definitivo que fueron derivados para SPECT de perfusión miocárdica sensibilizado con dipiridamol en enero de 2010 a junio de 2011; todos ellos implantados al menos 12 meses antes de la centellografía. La SPECT estuvo dirigida a evaluar la 
presencia y severidad de isquemia miocárdica a criterio del cardiólogo tratante. Se excluyeron los sujetos con estimulación endocárdica intermitente o ritmo sinusal en la adquisición de imágenes, gatillado dificultoso, índice de masa corporal $>35 \mathrm{~kg} / \mathrm{m}^{2}$, aneurisma ventricular izquierdo, valvulopatía mitro-aórtica severa, hipertensión arterial pulmonar al menos moderada, cardiopatía hipertrófica obstructiva, insuficiencia renal en hemodiálisis o problemas técnicos.

La SPECT de perfusión miocárdica se realizó en protocolo de 2 días (estrés-reposo), utilizando ${ }^{99 \mathrm{~m} T c-M I B I . ~ E n ~ l a ~ f a s e ~}$ de reposo se inyectó $20-25 \mathrm{mCi}(925-1,110 \mathrm{mBq})$ de ${ }^{99 \mathrm{~m}} \mathrm{Tc}$. A los 45-60 minutos se obtuvieron imágenes de SPECT gatillada con el electrocardiograma (ECG) mediante una gamma cámara de doble cabezal (GE Millenium VG), utilizando un colimador de baja energía y alta resolución. Se adquirieron imágenes en 30 paradas angulares cada 6 grados, de 25-30 segundos, a 8 imágenes/ciclo cardíaco. Para el gatillado se utilizó una ventana de intervalos R-R dentro del $20 \%$ de la longitud promedio del ciclo ECG previo al escaneado. Se siguieron las pautas de preparación, adquisición y procesamiento establecidas en las guías de la Asociación Europea de Medicina Nuclear. La presencia, topografía y severidad de los defectos de perfusión se evaluaron aplicando una escala visual semicuantitativa de 0-4 en un mapa polar de $17 \mathrm{seg}$ mentos, calculando los escores de suma en reposo (ESR) como indicadores de infarto. Además se obtuvieron: volumen sistólico y diastólico final del VI (VSF y VDF, en $\mathrm{ml}$ ) y $\mathrm{FEVI}$ en reposo (\%). La interpretación imagenológica fue realizada por 2 médicos nucleares expertos, resolviendo las diferencias por consenso. Teniendo en cuenta las imágenes tomográficas y los escores de suma, los expertos emitieron un juicio global diagnóstico sobre la presencia y severidad de la isquemia y/o infarto, diferenciando estos patrones de aquellos atribuibles a la estimulación por marcapasos.

Para el registro ECG de 12 derivaciones se utilizó un equipo digital (Exxer Inc., Argentina) a velocidad de $25 \mathrm{~mm} / \mathrm{seg}$ y voltaje de $10 \mathrm{~mm} / \mathrm{mV}$, con filtros de $5-40 \mathrm{~Hz}$. En un complejo P-QRS-T promediado (9 latidos) se midieron: frecuencia ventricular (cpm), intervalo PR (ms) y máxima duración de QRS (ms) en derivaciones V3-V4. La interpretación fue realizada por 2 cardiólogos clínicos, a ciegas de las imágenes.

Los pacientes fueron implantados de coordinación según indicación del cardiólogo clínico y electrofisiólogo tratantes y seleccionados para implante uni/bicameral siguiendo las recomendaciones actuales ${ }^{10}$. La ubicación del/los cables de estimulación endocárdica en la aurícula derecha y el VD (apical vs. tracto de salida) se definió mediante proyecciones estándar bajo control radioscópico. Todos los pacientes fueron controlados clínicamente y sometidos a interrogatorio telemétrico y ajustes de programación del dispositivo semestralmente, luego de un primer control postimplante. En el último control previo a la SPECT se registró la tasa de estimulación ventricular, redondeando en $5 \%$.

El método utilizado para extraer la amplitud -que representa el grado de engrosamiento sistólico de la pared del $\mathrm{VI}$ - y la fase -el momento de inicio de la contracción mecánica - a partir de los conteos regionales del VI y los cambios obtenidos en estos durante la SPECT ha sido descrito por Chen et al. ${ }^{11}$. Para el AF se aplicó SyncTool de Emory Cardiac Toolbox en su versión 3.02 HF, 2004 (Universidad de Emory, Syntermed, Atlanta). Esta herramienta asigna un ángulo de fase a cada píxel de la imagen de fase a partir del primer armónico de Fourier de la curva de actividad-tiempo. Del histograma de fase en reposo se obtuvieron desviación estándar $(D E F)$ y ancho de banda $(A B)$ en grados y se registró el segmento/s de inicio de la contracción del VI congelando las imágenes de vídeo-cine digital en el mapa polar a los $90-150^{\circ}$ de fase del inicio de la sístole. El procesamiento fue realizado a ciegas de los datos clínicos.

\section{Análisis estadístico}

Se presentan las estadísticas descriptivas de las variables clínicas y ECG, la tasa de estimulación ventricular (\%), ESR, VSF y VDF (ml) y FEVI en reposo (\%). Los promedios de los parámetros del AF se compararon con los valores de una población control ya reportada ${ }^{11}$ mediante test de «t» para muestras independientes con corrección de Welch. Los valores de DEF y $A B$ de las subpoblaciones de individuos con FEVI en reposo $\geq y<50 \%$ se compararon con los de la población control $^{11}$ mediante ANOVA de una vía y test de comparaciones múltiples (Tukey-Kramer). Para todos los análisis e utilizó el software Graphpad Instat ${ }^{\circledR}$, considerando como significativo un valor de $p<0.05$ ( 2 colas).

\section{Aprobación ética}

La investigación fue aprobada por el Comité de Ética institucional.

\section{Resultados}

Se estudiaron 12 pacientes mediante SPECT gatillada, 8 hombres $(67 \%)$, con rango etario de $62-84$ años (media $=75$ años), referidos por ángor (2/12), dolor torácico no anginoso $(3 / 12)$, disnea $(1 / 12)$, control de cardiopatía isquémica conocida o evaluación de otros síntomas sugestivos de isquemia $(6 / 12)$. Cuatro pacientes presentaban antecedentes de infarto, 2 de ellos habían sido sometidos a angioplastia coronaria y uno a cirugía de bypass coronario. En cuanto al tratamiento previo, 7 pacientes recibían fármacos inhibidores de la enzima conversora de angiotensina, 3 estaban bajo tratamiento bloqueador beta (casos 4, 6 y 7), 2 recibían nitritos de acción prolongada (casos 7 y 8 ) y 2 recibían agentes anticálcicos (casos 8 y 12).

El marcapasos definitivo estuvo indicado por BAVC sintomático en $9 / 12$ pacientes (75\%), habiendo transcurrido de 1.1 a 18.4 años (media $=4.9$ años) desde el implante. Un total de 10 pacientes fueron sometidos a implante bicameral. La tasa de estimulación ventricular fue $\geq 90 \%$ en todos los casos, excepto en la paciente 11, que mantenía ritmo sinusal en controles anuales previos. La contracción del VI se inició en el segmento septo-apical en 8/12 pacientes. Los hallazgos ECG basales y electrofisiológicos y el segmento de inicio de la contracción del VI se describen en la tabla 1, mientras que los resultados imagenológicos se resumen en la tabla 2. En opinión de expertos 4 pacientes tenían cicatriz de infarto, 3 de los cuales presentaban ESR $\geq 6$. El ESR promedio fue 9.2 ( $D E=6.3$, rango: $2-24)$. En cuanto a los volúmenes cavitarios basales los promedios (DE) fueron de $119.4 \mathrm{ml}$ (44.5) en el VDF y de $64.1 \mathrm{ml}(42.4)$ en el VSF. La $\mathrm{FEVI}$ en reposo fue de $51.2 \%(\mathrm{DE}=17.5 \%$, rango: $27-75 \%)$. 
Tabla 1 Variables ECG, electrofisiológicas y del análisis de fase

\begin{tabular}{|c|c|c|c|c|c|c|c|c|c|c|c|}
\hline \multirow[t]{2}{*}{$\mathrm{N}$} & \multicolumn{5}{|c|}{ ECG } & \multicolumn{5}{|l|}{ Electrofisiología } & \multirow{2}{*}{$\begin{array}{l}\text { AF } \\
\text { Segmento } \\
\text { inicio } \\
\text { contracción V }\end{array}$} \\
\hline & $\mathrm{S}$ & $\begin{array}{l}\text { Edad } \\
\text { (años) }\end{array}$ & $\begin{array}{l}\mathrm{FC} \\
(\mathrm{cpm})\end{array}$ & $\begin{array}{l}\text { Intervalo AV } \\
\text { programado } \\
(\mathrm{ms})\end{array}$ & $\begin{array}{l}\text { Duración } \\
\text { QRS (ms) }\end{array}$ & $\begin{array}{l}\text { Indicación } \\
\text { implante }\end{array}$ & $\begin{array}{l}\text { Tiempo desde } \\
\text { implante } \\
\text { (años) }\end{array}$ & $\begin{array}{l}\text { TEV } \\
(\%)\end{array}$ & Modo & $\begin{array}{l}\text { Sitio } \\
\text { cable } \\
\text { VD }\end{array}$ & \\
\hline 1 & $M$ & 75 & 65 & Var. & 143 & BAVC & 10.2 & 100 & VVI & Apical & Septo-apical \\
\hline 2 & M & 75 & 60 & 166 & 170 & BAVC & 18.4 & 95 & $\mathrm{DDD}$ & Apical & Septo-apical \\
\hline 3 & $\mathrm{~F}$ & 81 & 60 & 210 & 153 & BAVC sintomático & 1.0 & 100 & DDDR & $\begin{array}{l}\text { Septal } \\
\text { medio }\end{array}$ & Septal medio \\
\hline 4 & M & 68 & 75 & 160 & 183 & $\begin{array}{l}\text { Bloqueo } \\
\text { trifascicular } \\
\text { sintomático }\end{array}$ & 2.8 & 100 & $\mathrm{DDD}$ & $\begin{array}{l}\text { Septal } \\
\text { medio }\end{array}$ & Septo-apical \\
\hline 5 & M & 84 & 90 & 200 & 153 & BAVC & 1.1 & 100 & VDD & $\begin{array}{l}\text { Septal } \\
\text { medio }\end{array}$ & Septal medio \\
\hline 6 & M & 76 & 75 & 250 & 153 & $\begin{array}{l}\text { BAVC postablación } \\
\text { de flúter }\end{array}$ & 1.9 & 100 & VVIR & Apical & Septo-apical \\
\hline 7 & M & 70 & 70 & 216 & 186 & BAVC sintomático & 3.7 & 90 & DDD & Septal & Septo-apical \\
\hline 8 & M & 79 & 60 & 215 & 173 & $\begin{array}{l}\text { Síncope, BAVC } \\
\text { paroxístico }\end{array}$ & 1.6 & 95 & DDDR & $\begin{array}{l}\text { Septal } \\
\text { medio }\end{array}$ & Septo-apical \\
\hline 9 & M & 62 & 70 & 146 & 152 & BAVC sintomático & 1.0 & 100 & DDD & Apical & Septo-apical \\
\hline 10 & M & 74 & 70 & 160 & 166 & BAVC sintomático & 7.2 & 100 & DDD & Septal & Septal medio \\
\hline 11 & $\mathrm{~F}$ & 82 & 70 & 253 & 123 & $\begin{array}{l}\text { HSC y BAV } \\
\text { avanzado }\end{array}$ & 2.7 & $(*)$ & DDD & Apical & Septo-apical \\
\hline 12 & $\mathrm{~F}$ & 83 & 60 & 166 & 163 & BAVC sintomático & 7.3 & 100 & DDD & Apical & Septal medio \\
\hline
\end{tabular}

Variables del ECG basal, electrofisiológicas (del implante y el control telemétrico previo al SPECT) y del AF en reposo (segmento de inicio de la contracción del VI) en la serie de pacientes.

BAVC: bloqueo auriculoventricular completo; F: sexo femenino; FC: frecuencia cardíaca promedio; HSC: hipersensibilidad del seno carotídeo; M: sexo masculino; RS: ritmo sinusal; S: sexo; TEV: tasa de estimulación ventricular; Var.: variable latido a latido.

* Ritmo sinusal en el último año.

La figura 1 ilustra un paciente con imágenes de perfusión normal, FEVI conservada y AF normal. Se detectaron defectos de perfusión compatibles con infarto en el $60 \%$ de los sujetos que presentaban deterioro de la FEVI (pacientes 4, 6 y 7). Las imágenes obtenidas en el paciente 4 eran compatibles con infarto inferior, isquemia periinfarto moderada e isquemia remota en la región anterior, de intensidad moderada/severa. En este caso, la FEVI en reposo era de $33 \%$ y existía dilatación severa del VI. En el paciente 6, que presentaba antecedentes de infarto, se detectaron defectos

Tabla 2 Variables de interés en las imágenes de SPECT gatillado

\begin{tabular}{|c|c|c|c|c|c|c|c|}
\hline $\mathrm{N}$ & FEVI (\%) & ESR & Infarto & Infarto (extensión) & Isquemia (intensidad) & VDF (ml) & VSF $(\mathrm{ml})$ \\
\hline 1 & 58 & 15 & No & No & No & 81 & 34 \\
\hline 2 & 75 & 6 & No & No & No & 63 & 16 \\
\hline 3 & 27 & 14 & No & No & No & 139 & 101 \\
\hline 4 & 33 & 24 & Sí & Inferior basal y medio & Sí (M/S) & 216 & 144 \\
\hline 5 & 68 & 6 & No & No & No & 84 & 26 \\
\hline 6 & 34 & 6 & Sí & $\begin{array}{l}\text { Apical, septal basal y medio, } \\
\text { inferior, lateral basal y medio }\end{array}$ & No & 147 & 97 \\
\hline 7 & 27 & 6 & Sí & $\begin{array}{l}\text { Apical, inferior, septal basal } \\
\text { y medio, lateral basal }\end{array}$ & No & 163 & 120 \\
\hline 8 & 43 & 14 & No & No & No & 124 & 70 \\
\hline 9 & 59 & 2 & No & No & Sí (M) & 134 & 55 \\
\hline 10 & 56 & 6 & Sí & Inferior basal & No & 122 & 53 \\
\hline 11 & 64 & 6 & No & No & No & 89 & 32 \\
\hline 12 & 70 & 5 & No & No & No & 71 & 21 \\
\hline
\end{tabular}

El paciente 4 presentaba infarto asociado a isquemia periinfarto extensa y los pacientes 6 y 7 presentan defectos de perfusión compatibles con infarto extenso trasmural en territorio de la arteria coronaria derecha. En estos casos existía disfunción sistólica moderada/severa ( $F E V I<35 \%$ ) y dilatación del VI en reposo.

ESR: escore de suma en reposo; M/S: moderada/severa; VDF: volumen de fin de diástole del VI; VSF: volumen de fin de sístole del VI. 

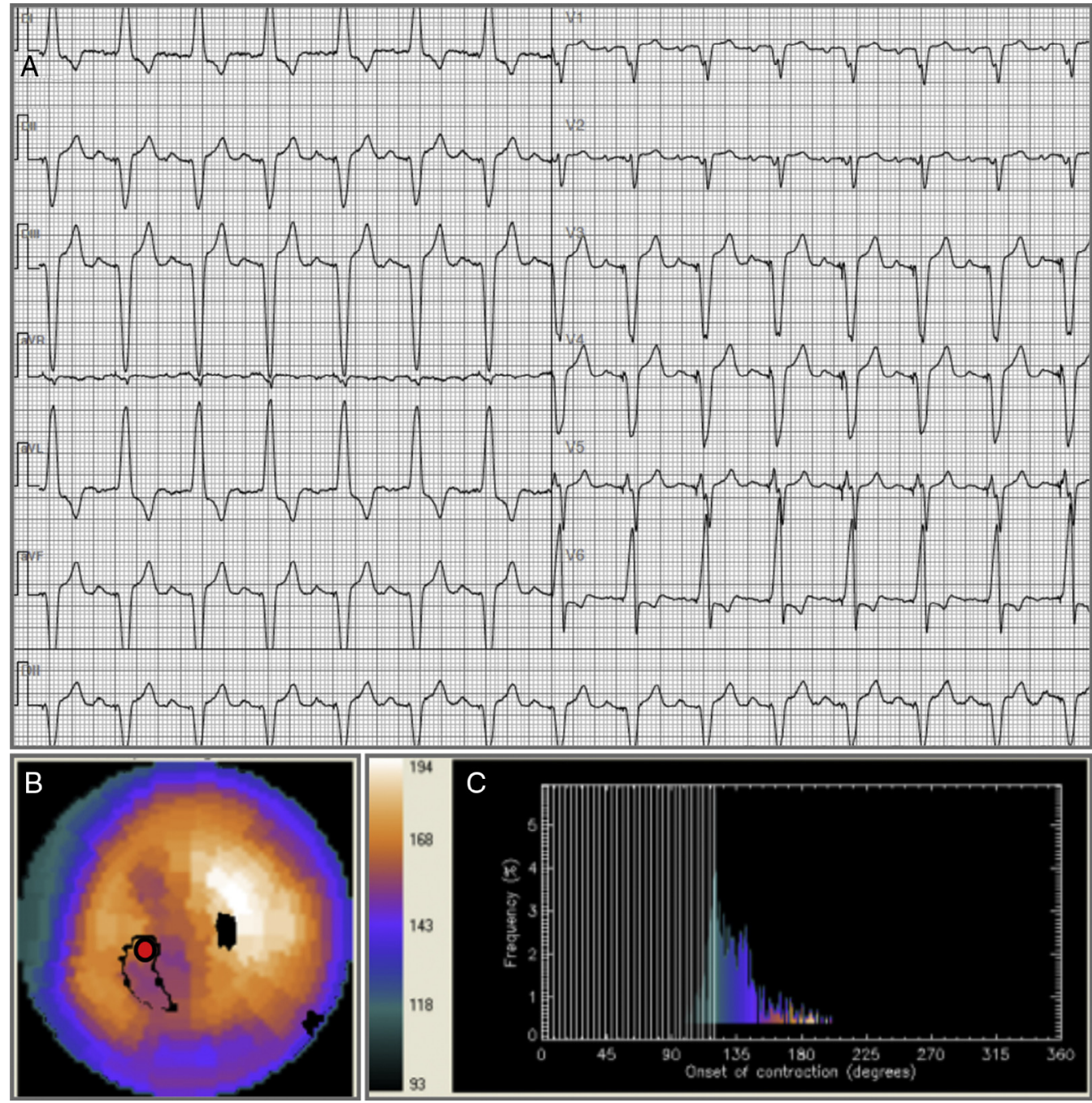

Figura 1 Análisis de fase en el caso 5 de la serie.

ECG basal (A), imágenes de vídeo-cine congeladas señalando el segmento de inicio de la contracción mecánica del VI (B) e histograma de fase en reposo (C). A. Estimulación endocárdica bipolar del ventrículo derecho a nivel apical. B. La contracción mecánica del VI se inicia a nivel septo-apical (círculo rojo). La distribución del radiotrazador en reposo es normal, $\mathrm{FEVI}=68 \%$. C. Aumento leve en el DEF y $A B$.

de perfusión compatibles con infarto transmural extenso (inferior y en segmentos apical, septal basal, septal medio, lateral basal y medio), FEVI en reposo de $34 \%$ y VI dilatado. En el caso 7 la SPECT gatillada mostró infarto extenso, disfunción sistólica severa y dilatación severa del VI (fig. 2). Los pacientes 9 y 10, ambos con función contráctil conservada, exhibían defectos de perfusión compatibles con isquemia de intensidad moderada e infarto localizados en el segmento inferior basal, respectivamente. No dispusimos de datos imagenológicos obtenidos antes del implante que permitan estimar mejor la evolución de los defectos de perfusión en respuesta a la estimulación endocárdica crónica.

Los casos de la serie exhibieron DMVI en el AF, evidenciada tanto por el DEF $\left(11.78 \pm 5.16^{\circ}\right.$ en controles ${ }^{11}$ vs. $\left.36.76 \pm 16.95^{\circ} ; p=0.0004\right)$ como el $A B\left(30.58 \pm 9.56^{\circ}\right.$ en controles vs. $\left.113.00 \pm 59.79^{\circ}\right)$. Tal como se ilustra en la figura 3 , los sujetos con $\mathrm{FEVI}<50 \%$ exhibieron mayor disincronía que aquellos con FEVI conservada/levemente disminuida (media $\mathrm{AB}=144.33^{\circ}$ en controles vs. $77.41^{\circ}, \mathrm{p}<0.001$; media $\mathrm{DEF}=46.67^{\circ}$ en controles vs. $\left.26.81^{\circ}, \mathrm{p}<0.01\right)$.

\section{Discusión}

Encontramos marcada DMVI en los individuos nuestra muestra, con una prevalencia superior a la descrita en otras series. En los sujetos con estimulación apexiana prolongada, la DMVI ha sido estudiada ampliamente utilizando doppler tisular ${ }^{12-15} \mathrm{y}$, en menor medida, mediante AF por SPECT gatillada. El doppler tisular detecta DMVI hasta en el $57 \%$ de los pacientes con ablación del haz de His y estimulación apical ${ }^{15}$. Aplicando AF por SPECT gatillada en sujetos con diferentes trastornos de la conducción ventricular Trimble et al. confirman que existe marcada DMIV en los pacientes con marcapasos, con valores de $30.81 \pm 9.40^{\circ}$ y $94.10 \pm 62.7^{\circ}$ en el DEF y el $A B$, respectivamente ${ }^{16}$. Este reporte no describe la ubicación del cable en el VD, la tasa de estimulación ni otros parámetros electrofisiológicos. 

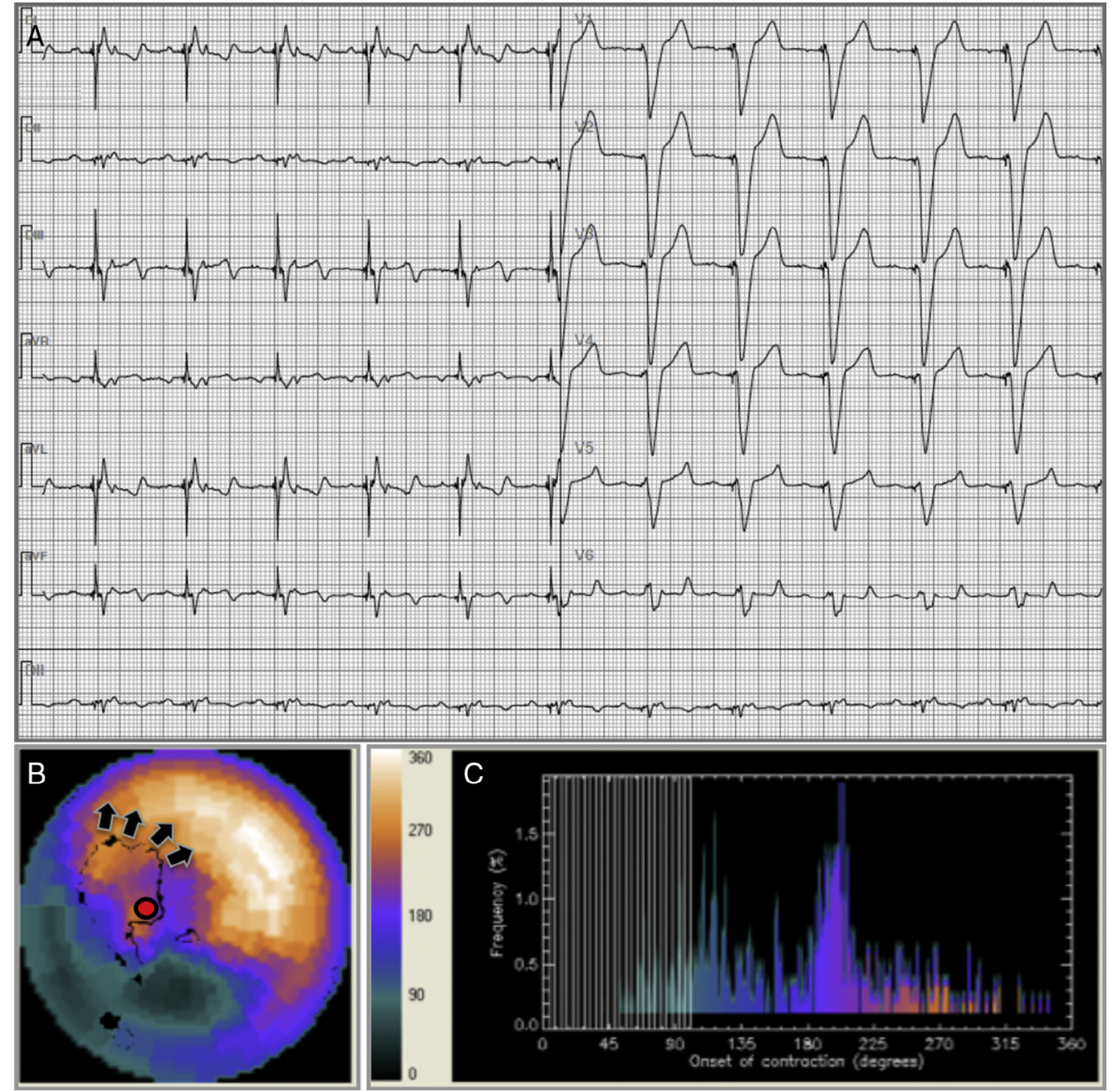

Figura 2 Análisis de fase el caso 7 de la serie.

A. Estimulación endocárdica bipolar apical, zona inactivable inferior. B. La contracción del VI se inicia en el segmento septo-apical (círculo rojo) y se expande luego al segmento antero-septal medio (flechas negras). Existe un defecto de captación del radiofármaco compatible con infarto inferior que se extiende a la base del septum. Tras la interpretación de las imágenes estrés-reposo no se detectó isquemia circundante al área cicatricial. $\mathrm{FEVI}$ en reposo $=27 \%$, VSF $=120 \mathrm{ml}$. C. DEF y $A B$ muy prolongados en el histograma de fase en reposo.

Modificado de: Fernando-Castagnetto et al. $^{30}$.

La DMVI se observó en tiempos variables desde el implante (1.1-18.4 años, media: 4.9 años). El único individuo con baja tasa de estimulación presentaba duración del QRS de $124 \mathrm{~ms}$, FEVI en reposo y perfusión normales (caso 11). Este caso, una mujer de 82 años implantada por síndrome de hipersensibilidad del seno carotídeo sintomático con bloqueo aurículo-ventricular avanzado, fue el único de la serie que exhibió un histograma de fase en reposo normal $\left(\mathrm{DEF}=11.78^{\circ}, \mathrm{AB}=40^{\circ}\right)$. Aunque los hallazgos experimentales en animales sugieren que la DMVI podría instalarse tempranamente tras el implante de marcapasos ${ }^{17}$, en humanos no está claro si este comportamiento existe desde el inicio de la estimulación endocárdica o, por el contrario, si se instala a más largo plazo. Parece existir una relación directa entre la tasa y el modo de estimulación ventricular con algunos resultados clínicos adversos. Un subestudio del Mode Selection Trial detectó una fuerte asociación entre la tasa de estimulación y el riesgo de hospitalización por insuficiencia cardíaca y fibrilación auricular ${ }^{12}$. El estudio DAVID evidenció que la estimulación en modo VVI se asociaba con mayor mortalidad y tasa de ingresos por insuficiencia cardíaca que el modo bicameral secuencial ${ }^{18}$; las mayores diferencias en la incidencia de eventos adversos entre ambos modos se apreciaron con una tasa de estimulación ventricular acumulativa $\geq 40 \%{ }^{19}$. Aun cuando estas investigaciones no estuvieron dirigidas a definir la tasa de estimulación ventricular «crítica» a partir de la cual podría aumentan el riesgo de eventos adversos, estos hallazgos sientan las bases para recomendar la estimulación biventricular en los pacientes con estimulación del VD y disfunción sistólica ${ }^{20}$.

La estimulación prolongada septal tendió a asociarse con una DMVI más acentuada que la estimulación apical, aunque no fue posible testear una asociación estadística. Las 


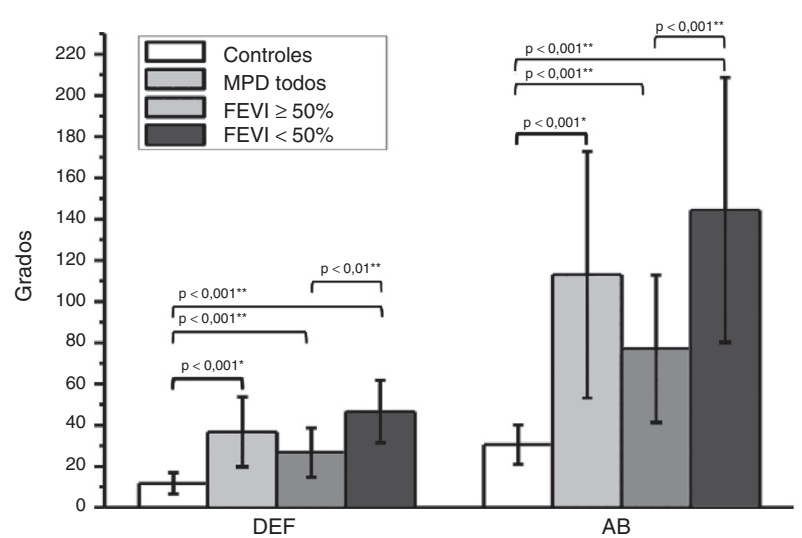

Figura 3 Comparación del análisis de fase con controles y entre subgrupos.

Los gráficos de barras comparan el DEF y el $A B$ entre los pacientes de una población normal (controles) ${ }^{11}$ con los pacientes de la serie analizada completa (MP todos) y con las subpoblaciones de pacientes con $\mathrm{FEVI} \geq \mathrm{y}<50 \%$.

MP: marcapasos.

"Test de «t» para muestras independientes con corrección de Welch.

${ }^{* *}$ ANOVA de una vía y test de comparaciones múltiples (TurkeyKramer) grupo control-subgrupos con FEVI $\geq \mathrm{y}<50$.

experiencias con doppler tisular sugieren que la estimulación prolongada a nivel del haz de His («fisiológica») y la pared septal del tracto de salida del VD podrían determinar menor DMVI que la estimulación apical ${ }^{21,22}$, pero los efectos diferenciales sobre los eventos adversos son inciertos. Nos sorprendió encontrar que la estimulación en el septum del VD se asociara con una mayor duración del complejo QRS que la estimulación apical en nuestra serie, lo que podría explicarse por el pequeño número de la muestra y la selección de esta por conveniencia. Por otro lado, las investigaciones que compararon la DMVI asociada a distintos sitios de estimulación mediante AF por SPECT gatillada son muy recientes. Zhang et al. compararon la DMVI en pacientes portadores de BAV completo con estimulación apical $(n=20)$ vs. septal $(n=19)$, comprobando que la estimulación apical se asoció con mayor DMVI a los 6 meses del implante ${ }^{23}$. Sin embargo, un estudio prospectivo observacional con una muestra mayor de pacientes $(n=51)$ no encontró diferencias en la disincronía a los 12 meses de seguimiento ${ }^{24}$.

Es posible que los efectos negativos de la estimulación endocárdica por marcapasos sean mayores en la cardiopatía isquémica con trastornos de la conducción ventricular al momento del implante que en otras cardiopatías. En nuestra serie el $60 \%$ de los individuos con deterioro de la FEVI evidenciaban defectos de perfusión compatibles con infarto extenso y/o isquemia severa. Al estudiar 125 pacientes con $\mathrm{FEVI} \leq 35 \%$ (15.2\% de ellos con marcapasos), Varma et al. observaron que los índices $D E F$ y $A B$ se correlacionaban con isquemia severa y duración más prolongada del $\mathrm{QRS}^{25}$. Incluso algunas evidencias sugieren que los individuos con cicatriz de infarto pueden exhibir marcada DMVI en ausencia de ensanchamiento del QRS. Estos datos surgen de investigaciones que aplicaron tanto doppler $\operatorname{tisular}^{26}$ como AF por SPECT gatillada ${ }^{27}$. Basándonos en nuestros hallazgos resulta muy difícil definir cuáles son los cambios atribuibles a la propia estimulación del VD y cuáles se deben a la enfermedad de base. Como las imágenes procesadas se refieren a la perfusión del VI en condiciones basales (sin ejercer estrés farmacológico), es poco probable que en nuestros casos la disincronía se asocie con la presencia de isquemia significativa al momento de adquirir las imágenes, aunque sí con la presencia y extensión del miocardio infartado (cicatriz). El diseño observacional transversal y el tamaño muestral reducido de nuestra experiencia, que observó a unos pocos pacientes puntualmente al momento de la SPECT, hizo imposible conocer las maniobras terapéuticas que se hicieron para resolver la isquemia y su potencial influencia sobre el comportamiento de los parámetros de volumen y disincronía. Esto podría evaluarse mediante un diseño de cohortes con un número mayor de pacientes. En realidad, la evaluación de la isquemia, la hibernación o el atontamiento miocárdico repetido sobre la DMVI en los pacientes con marcapasos sigue siendo un área poco explorada. Si uno pensara en la DMVI asociada a estos fenómenos metabólicos como un estado contráctil potencialmente reversible, la estimulación por marcapasos podría mantener y/o exacerbar ciertas heterogeneidades en la perfusión miocárdica que ya están presentes al implante ${ }^{28} \mathrm{y}$, quizás, acarrear un riesgo elevado de infarto y deterioro funcional que sería pasible de intervenciones coronarias. Desafortunadamente, los estudios disponibles fueron diseñados para predecir la respuesta a la TRC en pocos pacientes con marcapasos, y no han analizado el impacto de diferentes intervenciones sobre el territorio isquémico ${ }^{29}$.

\section{Limitaciones}

A pesar de tratarse de un ensayo piloto, la mayor limitación de nuestra experiencia está signada por el escaso número de pacientes de nuestra serie, que representan apenas el $0.3 \%$ de los estudios de perfusión sensibilizados con dipiridamol. Las dificultades que existen para el gatillado en los pacientes con ritmo de marcapasos redujeron más aún la muestra. El escaso tamaño muestral y el diseño transversal impidieron analizar otras variables de interés clínico y fisiológico, como la relación de la DMVI con la ubicación exacta del sitio de estimulación en el tracto de salida del VD, o la duración y morfología del QRS intrínseco previo al implante y en la evolución, por ejemplo. En segundo lugar, evaluamos la DMVI en sujetos añosos con probabilidad pretest elevada para cardiopatía isquémica, lo que imprime un sesgo de indicación y limita la extrapolación de los resultados. En tercer lugar, existieron dos obstáculos inherentes a la técnica de SPECT: las limitaciones para diagnosticar isquemia/infarto en el territorio de la arteria descendente anterior debido al BCRI artificial y la elevada tasa de defectos de perfusión falsos positivos asociados a la estimulación por marcapasos ${ }^{5}$. Intentamos atacar estos problemas incluyendo la opinión de expertos en la interpretación de las imágenes y descartando los individuos con artefactos de atenuación por obesidad. Finalmente, no fue posible contrastar los hallazgos centellográficos con la angiografía coronaria.

\section{Potenciales implicaciones clínicas}

Es posible que para evaluar la contribución de diversos factores a la DMVI como la etiología de las alteraciones 
estructurales, la FEVI o el estado del sistema de conducción ventricular previo al implante, por ejemplo, se requiera información provista por más de una modalidad diagnóstica de imagen. El AF por SPECT gatillada es una técnica que informa sobre la dinámica contráctil de toda la masa del $\mathrm{VI}$, se correlaciona bien con el doppler tisular, se aplica sobre las imágenes crudas de la SPECT y automatiza el procesamiento de las imágenes con escasa variabilidad ${ }^{9}$. Considerando que la información que provee el AF sobre la heterogeneidad temporal contráctil se agrega a las variables funcionales convencionales provistas por la SPECT gatillada, se comprende que esta técnica puede aportar más al conocimiento de la disincronía en la cardiopatía isquémica que en otras etiologías. En otras cardiopatías subyacentes (valvulares aórticas, miocardiopatías dilatadas no isquémicas, cardiopatía hipertrófica, etc.) el doppler tisular exhibe bondades adicionales en manos experimentadas que se derivan de su mayor resolución espacial y su capacidad de evaluar en un solo estudio múltiples variables anatómicas y fisiológicas asociadas a otras disincronías (interventricular y auriculoventricular).

En teoría, la indicación oportuna del pasaje (upgrading) a TRC podría desacelerar la evolución adversa asociada a la estimulación prolongada por marcapasos ${ }^{6}$. Por desgracia, e incluso con base en las recomendaciones actuales ${ }^{20}$, estos beneficios deben esperar la oportunidad de un estado de deterioro anatómico y funcional del VI considerable, a veces irreversible. Pensamos que la influencia de la masa y topografía del miocardio necrótico y la cuantía del miocardio hibernado sobre la progresión al deterioro de la FEVI en los sujetos con marcapasos merecería ser evaluada en profundidad. En este escenario podría cobrar importancia un análisis individualizado de la DMVI con antelación al implante o cuando se considere el pasaje a TRC.

A pesar de las limitaciones inherentes a nuestra investigación, un ensayo piloto unicéntrico que aplicó una técnica nueva en pacientes muy seleccionados, pensamos que los resultados obtenidos pueden estimular a ampliar la serie de estudio. En efecto, de confirmarse en experiencias mayores, nuestros hallazgos podrían generar la hipótesis de que la tasa de estimulación ventricular y la masa de infarto son variables, que ejercen diferente peso relativo sobre la DMVI que otros factores, como el sitio de estimulación en el VD o el grado de disincronía auriculoventricular, por ejemplo.

\section{Conclusiones}

En la cardiopatía isquémica una tasa de estimulación endocárdica del VD elevada se asocia con marcada DMVI, un hallazgo que está presente incluso cuando la función sistólica está conservada. En los pacientes con coronariopatía avanzada que requieren estimulación endocárdica prolongada del VD, el AF por SPECT gatillada podría ser una herramienta útil para evaluar la disincronía asociada al infarto, e incluso para decidir oportunamente el pasaje (upgrading) al modo biventricular.

\section{Responsabilidades éticas}

Protección de personas y animales. Los autores declaran que para esta investigación no se han realizado experimentos en seres humanos ni en animales.
Confidencialidad de los datos. Los autores declaran que han seguido los protocolos de su centro de trabajo sobre la publicación de datos de pacientes.

Derecho a la privacidad y consentimiento informado. Los autores declaran que en este artículo no aparecen datos de pacientes.

\section{Financiación}

No se recibió patrocinio de ningún tipo para llevar a cabo este artículo.

\section{Conflicto de intereses}

Los autores declaran no tener ningún conflicto de intereses.

\section{Agradecimientos}

Al Programa de Investigación Biomédica (Pro.In.Bio) de la Facultad de Medicina - Universidad de la República, que proveyó apoyo parcial para el desarrollo de esta investigación en el marco de un proyecto de Maestría.

Al Dr. Adrián Baranchuk, por sus valiosos aportes en la revisión del manuscrito.

\section{Bibliografía}

1. Saleh S, Liakopoulos OJ, Buckberg GD. The septal motion of biventricular function. Eur J Cardio-Thorac Surg. 2006;29S:S126-38.

2. Cheng A, Helm RH, Abraham TP. Pathophysiological mechanisms underlying ventricular dyssynchrony. Europace. 2009;11:v10-4.

3. Auricchio A, Fantoni C, Regoli F, et al. Characterization of left ventricular activation in patients with heart failure and left bundle-branch block. Circulation. 2004;109:1133-9.

4. Prinzen FW, Hunter WC, Wyman BT, et al. Mapping of regional myocardial strain and work during ventricular pacing: Experimental study using magnetic resonance imaging tagging. J Am Coll Cardiol. 1999;33:1735-42.

5. Tse HF, Lau CP. Long-term effect of right ventricular pacing on myocardial perfusion and function. J Am Coll Cardiol. 1997;29:744-9.

6. Tops LF, Schalij MJ, Bax JJ. The effects of right ventricular apical pacing on ventricular function and dyssynchrony implications for therapy. J Am Coll Cardiol. 2009;54:764-76.

7. Sweeney MO, Hellkamp AS. Heart failure during cardiac pacing. Circulation. 2006;113:2082-8.

8. Nielsen JC. Mortality and incidence of atrial fibrillation in paced patients. J Cardiovasc Electrophysiol. 2002;13 1 Suppl:S17-22.

9. Trimble MA, Borges-Neto S, Velazquez EJ, et al. Emerging role of myocardial perfusion imaging to evaluate patients for cardiac resynchronization therapy. Am J Cardiol. 2008;102:211-7.

10. Epstein AE, DiMarco JP, Ellenbogen KA, et al. American College of Cardiology Foundation; American Heart Association Task Force on Practice Guidelines; Heart Rhythm Society. 2012 ACCF/AHA/HRS focused update incorporated into the ACCF/AHA/HRS 2008 guidelines for device-based therapy of cardiac rhythm abnormalities: A report of the American College of Cardiology Foundation/American Heart Association Task Force on Practice Guidelines and the Heart Rhythm Society. Circulation. 2013;127:e283-352. 
11. Chen J, Garcia EV, Folks RD, et al. Onset of left ventricular mechanical contraction as determined by phase analysis of ECGgated myocardial perfusion SPECT imaging: Development of a diagnostic tool for assessment of cardiac mechanical dyssynchrony. J Nucl Cardiol. 2005;12:687-95.

12. Sweeney MO, Hellkamp AS, Ellenbogen KA, et al. Adverse effect of ventricular pacing on heart failure and atrial fibrillation among patients with normal baseline QRS duration in a clinical trial of pacemaker therapy for sinus node dysfunction. Circulation. 2003;107:2932-7.

13. Thambo JB, Bordachar P, Garrigue S, et al. Detrimental ventricular remodeling in patients with congenital complete heart block and chronic right ventricular apical pacing. Circulation. 2004; 110:3766-72.

14. Schmidt M, Brömsen J, Herholz C, et al. Evidence of left ventricular dyssynchrony resulting from right ventricular pacing in patients with severely depressed left ventricular ejection fraction. Europace. 2007;9:34-40.

15. Tops LF, Suffoletto MS, Bleeker GB, et al. Speckle-tracking radial strain reveals left ventricular dyssynchrony in patients with permanent right ventricular pacing. J Am Coll Cardiol. 2007;50:1180-8

16. Trimble MA, Borges-Neto S, Smallheiser $S$, et al. Evaluation of left ventricular mechanical dyssynchrony as determined by phase analysis of ECG-gated myocardial perfusion SPECT imaging in patients with left ventricular dysfunction and conduction disturbances. J Nucl Cardiol. 2007;14:298-307.

17. Badke FR, Boinay P, Covell JW. Effects of ventricular pacing on regional left ventricular performance in the dog. Am J Physiol. 1980;238:H858-67.

18. Wilkoff BL1, Cook JR, Epstein AE, et al., on behalf of the Dual Chamber and VVI Implantable Defibrillator Trial Investigators. Dual chamber pacing or ventricular backup pacing in patients with an implantable defibrillator: The Dual Chamber and VVI Implantable Defibrillator (DAVID) trial. JAMA. 2002;288:3115-23.

19. Sharma AD, Rizo-Patron C, Hallstrom AP, et al., DAVID Investigators. Percent right ventricular pacing predicts outcomes in the DAVID trial. Heart Rhythm. 2005;2:830-4.

20. Russo AM, Stainback RF, Bailey SR, et al. ACCF/HRS/AHA/ASE/ HFSA/SCAI/SCCT/SCMR 2013 appropiate use criteria for implantable cardioverter-defibrillators and cardiac resynchronization therapy: a report of the American College of Cardiology Foundation; Appropiate Use Criteria Task Force, Heart Rhythm Society, American Heart Association, American Society of
Echocardiography, Heart Society of America, Society for Cardiovascular Angiography and Interventions, Society of Cardiovascular Computed Tomography, and Society for Cardiovascular Magnetic Resonance. J Am Coll Cardiol. 2013;61:1323-73.

21. Manolis A. The deleterious consequences of right ventricular apical pacing: Time to seek alternate site pacing. PACE. 2006;29:298-315.

22. Catanzariti D, Maines M, Cemin C, et al. Permanent direct his bundle pacing does not induce ventricular dyssynchrony unlike conventional right ventricular apical pacing. An intrapatient acute comparison study. J Interv Card Electrophysiol. 2006;16:81-92.

23. Zhang $H$, Hou $X$, Wang $Y$, et al. The acute and chronic effects of different right ventricular site pacing on left ventricular mechanical synchrony as assessed by phase analysis of SPECT myocardial perfusion imaging. J Nucl Cardiol. 2014;21: 958-66.

24. Singh H, Patel CD, Sharma G, et al. Comparison of left ventricular systolic function and mechanical dyssynchrony using equilibrium radionuclide angiography in patients with right ventricular outflow tract versus right ventricular apical pacing: A prospective single-center study. J Nucl Cardiol. 2015;22:903-11.

25. Varma N. Left ventricular conduction delays induced by right ventricular apical pacing: Effect of left ventricular dysfunction and bundle branch block. J Cardiovasc Electrophysiol. 2008;19:114-22.

26. Zhang Y, Chan AK, Yu CM, et al. Left ventricular systolic asynchrony after acute myocardial infarction in patients with narrow QRS complexes. Am Heart J. 2005;149:497-503.

27. Murro J, Esteves F, Galt J, et al. Characterization of mechanical dyssynchrony measured by gated single photon emission computed tomography phase analysis after acute ST-elevation myocardial infarction. J Nucl Cardiol. 2011;18:912-9.

28. Lindner O, Vogt J, Kammeier A, et al. Effect of cardiac resynchronization therapy on global and regional oxygen consumption and myocardial blood flow in patients with non-ischaemic and ischaemic cardiomyopathy. Eur Heart J. 2005;26:70-6.

29. Sciagra R, Giaccardi M, Porciani MC, et al. Myocardial perfusion imaging using gated SPECT in heart failure patients undergoing cardiac resynchronization therapy. J Nucl Med. 2003;45: 164-8.

30. Ferrando-Castagnetto F, Ricca-Mallada R, Vidal A, et al. Disincronía ventricular izquierda. en la estimulación septal prolongada. Medicina (Buenos Aires). 2016;76:321-5. 